\title{
Cinética da secagem do feijão verde (Vigna unguiculata $L$. Walp)em micro-ondas com e sem pré-tratamento osmótico
}

\author{
A.L.M.L. da MATA ${ }^{1}$, S.E.D. COSTA $^{1}$, D. P. CAPISTRANO ${ }^{1}$, F.C. de MORAES FILHO ${ }^{1}$
}

${ }^{1}$ Universidade Federal do Rio Grande do Norte, Departamento de Engenharia Química E-mail para contato: anadamata@eq.ufrn.br

\begin{abstract}
RESUMO - A secagem é uma das mais antigas e comuns operações unitárias utilizadas nos mais diversos processos dos mais diferentes tipos de indústrias.O estudo da mesma é de grande relevância principalmente para indústria alimentícia, uma vez que a secagem é um dos processos mais utilizados para a preservação de alimentos, pois promove a retirada de grande parte da água livre do alimento, minimizando a ocorrência de reações de deterioração química e dos ataques de micro-organismos.A desidratação osmótica é a desidratação parcial do alimento, o qual é mergulhado em uma solução hipertônica para que haja o processo de transferência de massa.A utilização da tecnologia de micro-ondas se mostra mais eficiente na realização da secagem, pois, o tempo de realização dessa operação é bastante reduzido em comparação a outros métodos, o que motiva o seu uso. Este trabalho tem como principal objetivo a modelagem matemática do processo de secagem de feijão verde (Vigna unguiculata L. Walp) in natura e com desidratação osmótica, utilizando a tecnologia de micro-ondas.Os dados experimentais da cinética de secagem foram ajustados pelas equações de Page, Lewis, Page Modificado, Henderson \& Pabis e Equação Simplificada da Difusão de Fick (ESDF). O tempo de secagem para as amostras que passaram pelo prétratamento, desidratação osmótica, mostrou-se menor, indicando uma economia energética e maior eficiência do processo. O modelo mais representativo para as secagens foi o de Page.
\end{abstract}

\section{INTRODUÇÃO}

A secagem é uma das mais antigas e comuns operações unitárias utilizadas nos mais diversos processos dos mais diferentes tipos de indústrias (Menezes et al., 2010). Esta técnica consiste na retirada de grande parte da água livre do alimento, permitindo o seu transporte e armazenamento a um custo relativamente baixo, porém apresenta o inconveniente de causar danos ao mesmo, tais como perdas de vitaminas e de textura (Azoubel et al., 2010), baixa eficiência energética e elevado tempo de secagem (Feng e Tang, 1998).

A utilização da tecnologia de micro-ondas se mostra como uma possível alternativa para a realização da secagem, pois, o tempo de realização dessa operação é bastante reduzido em comparação a outros métodos, o que motiva o seu uso(Oliveira et al., 2009). 
O aquecimento por micro-ondas é efetuado por radiações sobre as moléculas de água que se aquecem pela oscilação a altas frequências. Esse aquecimento seletivo (aliado à secagem rápida) promove menor perda de componentes voláteis do que em estufas convencionais (Da Luz et al., 1998).

A desidratação osmótica (DO) pode ser descrita como uma desidratação parcial de alimentos através do processo de osmose, o qual envolve a imersão do alimento por um dado período de tempo em uma solução hipertônica normalmente de sal e/ou açúcar. (Rastogi et al., 2004; Piotrowski et al., 2004).

O pré-tratamento osmótico efetuado antes da secagem tem a função de minimizar os impactos negativos da mesma, como perda de aroma, escurecimento enzimático e perda da cor natural dos alimentos. Além disso, em alguns produtos, fatores adicionais como capacidade e velocidade de reidratação devem ser considerados. Em função da aplicação e uso do alimento desidratado o objetivo da desidratação é obter produtos que, quando reconstituídos, apresentem textura adequada o mais rápido possível (Sanjuán et al., 1999; Moreira et al., 2008; Krokida e Philippopoulos, 2005).

Durante a desidratação algumas mudanças importantes ocorrem no alimento, tais como modificações estruturais e físico-químicas que são influenciadas pela composição das amostras, pelas condições impostas durante a secagem, e por qualquer pré-tratamento ao qual o produto tenha sido submetido, alterando consequentemente a qualidade do produto final (Maskan, 2001).

A qualidade do produto está cada vez mais sendo exigida nos processos de desidratação de alimentos. A desidratação osmótica antes do processo de secagem melhora as propriedades nutricionais, sensoriais e funcionais do alimento sem alterar sua integridade. Melhora também a textura, a estabilidade da coloração durante a posterior secagem e o armazenamento do produto desidratado. Além disso, em alguns produtos, fatores adicionais como capacidade e velocidade de reidratação devem ser considerados. Em função da aplicação e uso do alimento desidratado, o objetivo da desidratação é obter produtos que, quando reconstituídos, apresentem textura adequada o mais rápido possível (Sanjuán et al., 1999; Moreira et al., 2008; Krokida e Philippopoulos, 2005).

O feijão verde (Vigna unguiculata L. Walp) é uma cultura de grande importância socioeconômica nas regiões Norte e Nordeste do Brasil. Sua produção constitui-se numa importante fonte de renda para os agricultores familiares e tem um grande potencial para a expansão do consumo, assim como também para o processamento industrial.

O estudo da cinética de secagem do feijão verde pode ser bastante útil para a otimização do processo. Do ponto de vista de processamento e engenharia, é interessante conhecer quão rápida é a perda de água e avaliar a adequação de diferentes equações para a descrição da cinética do processo (Santos e Prado, 2010).

Este trabalho tem como principal objetivo a modelagem matemática do processo de secagem de feijão verde (Vigna unguiculata L. Walp) in natura e com desidratação osmótica, utilizando a tecnologia de micro-ondas.

\section{MATERIAIS E MÉTODO}


As amostras de feijão verde (Vigna unguiculata L. Walp) foram compradas em mercado local, em Natal/RN. A seleção das amostras foi realizada separando-se os grãos mais verdes de aspecto intacto.

\subsection{Desidratação Osmótica}

Foram preparadasamostras do feijão verde para o processo de secagem com Desidratação Osmótica (OD).Para a realização do pré-tratamento osmótico, utilizou-se uma solução aquosa de $\mathrm{NaCl}$ de concentração mássica igual a 12,5\%. Essa etapa foi desenvolvida com uma proporção de feijão-solução de $1: 3$, à temperatura de $40^{\circ} \mathrm{C}$, controlada em banhomaria, sem agitação, durante 20 minutos. Após esse tempo, as amostras foram deixadas sobre um papel toalha por 5 minutos para remover o excesso da solução. As condições do prétratamento osmótico foram estabelecidas em estudos anteriores (Oliveira et al., 2009).

\subsection{Secagem em Micro-ondas}

A secagem de todas as amostras de feijão verde foi realizada em um micro-ondas (2451 $\mathrm{MHz}$ e $1,7 \mathrm{~kW}$ ) adaptado com uma saída de ar ligada a um compressor. Sem esta modificação, o ar dentro do micro-ondas ficaria saturado com vapor d'água e a secagem seria prejudicada.

Foram realizadas secagens-testes para se determinar a massa inicial das amostras e o tempo de secagem mais adequado.Para o feijão verde in natura,as secagens foram de 30 segundos no forno micro-ondas, intercaladas por intervalos de tempo para pesagem das amostras em balança analítica. As massas iniciais (Mi) foram de 50, 40 e 30 gramas, respectivamente. Estas secagens foram finalizadas quando a massa constante foi obtida. $\mathrm{O}$ aspecto físico dos grãos após as secagens foi imprescindível na escolha da massa inicial que seria considerada para a modelagem da cinética da secagem, pois o mesmo é determinante no mercado consumidor.Desta forma, a massa de $30 \mathrm{~g}$ foi adotada como a inicial.

Para as amostras com OD, foram realizadas análises semelhantes e a massa inicial também foi de $30 \mathrm{~g}$, porém,o tempo ótimo de secagem encontrado foi de $15 \mathrm{~s}$, pois muitos grãos apresentaram aspecto indesejado com tempo de secagem igual a 30s.

\subsection{Modelos Matemáticos de Curvas de Secagem}

Os dados experimentais da cinética de secagem devem ser ajustados pelas equações dePage (Equação 1), Lewis (Equação 2), Page Modificado (Equação 3), Henderson \& Pabis (Equação 4)e Equação Simplificada da Difusão de Fick (ESDF) (Equação 5).

Equações:

$$
\begin{aligned}
& M R=\exp \left(-k \cdot\left(t^{n}\right)\right) \\
& M R=\exp (-k \cdot t) \\
& M R=\exp (-k \cdot t)^{n} \\
& M R=\text { a.exp }(-k \cdot t)
\end{aligned}
$$


Onde:

$$
M R=\text { a. } \exp \left(-c \cdot\left(\frac{t}{L^{2}}\right)\right)(5)
$$

MR - razão de umidade (adimensional);

$\mathrm{k}, \mathrm{t}, \mathrm{n}, \mathrm{a}, \mathrm{L}-$ constantes dos modelos;

$\mathrm{t}$-tempo (s).

A razão de umidade (MR) é definida pela Equação 6.

$$
M R=(\mathrm{Mt}-\mathrm{Me}) /(\mathrm{Mo}-\mathrm{Me})(6)
$$

Onde,

Mt - umidade no tempo t;

Mo - umidade inicial;

$\mathrm{Me}$ - umidade de equilíbrio.

Parâmetros de análise estatística: Ocoeficiente de correlação $\left(\mathrm{R}^{2}\right)$, o erro viés principal (MBE), o erro médio quadrático (RMSE) e o Qui quadrado reduzido $\left(\chi^{2}\right)$ tem sido utilizados para avaliar o desempenho dos modelos de secagem (Gunhan et al., 2005).

O R $\mathrm{R}^{2}$ é utilizado para testar a relação linear entre os valoresmedidos e os estimados.Ele é calculado pela Equação 7 .

$$
R^{2}=\frac{\sum_{\mathrm{i}=1}^{\mathrm{N}}(\mathrm{MRi}-\mathrm{MRpre}, \mathrm{i}) \cdot(\mathrm{MRi}-\mathrm{MRexp}, \mathrm{i})}{\sqrt{\left(\sum_{\mathrm{i}=1}^{\mathrm{N}}(\mathrm{MRi}-\mathrm{MRpred}, \mathrm{i})^{2}\right) \cdot\left(\sum_{\mathrm{i}=1}^{\mathrm{N}}(\mathrm{MRi}-\mathrm{MRexp}, \mathrm{i})^{2}\right)}}
$$

Onde,

MRi - razão de umidade inicial;

MRexp,i - razão de umidade encontrada no ponto i;

MRpre, i - razão de umidade calculada no ponto i;

$\mathrm{N}$ - Número de pontos.

O MBE fornece informações sobre o desempenho de longo prazo das correlações, permitindo uma comparação do desvio real entre os valores preditos e medidos, termo a termo. O mesmo é dado pela Equação 8.

$$
M B E=\frac{\sum_{\mathrm{i}=1}^{\mathrm{N}}(\text { MRpre }, \mathrm{i}-\mathrm{MRexp}, \mathrm{i})}{\mathrm{N}}
$$

O RMSE fornece informações sobre o desempenho de curto prazo das correlações e é definido pela Equação 9.

$$
R M S E=\left(\frac{\sum_{\mathrm{i}=1}^{\mathrm{N}}(\mathrm{MRpre}, \mathrm{i}-\mathrm{MRexp}, \mathrm{i})}{\mathrm{N}}\right)^{\frac{1}{2}}
$$

O $\chi^{2}$ é definido pela Equação 10.

$$
\chi^{2}=\frac{\sum_{\mathrm{i}=1}^{\mathrm{N}}(\mathrm{MRexp}, \mathrm{i}-\mathrm{MRpred}, \mathrm{i})^{2}}{\mathrm{~N}-\mathrm{n}}
$$


Onde,

$\mathrm{n}$ - número de constantes do modelo.

Quanto mais próximo de 1 for o valor do $\mathrm{R}^{2}$ e de 0 forem os dos demais parâmetros, melhor será o ajuste do modelo.

As modelagens matemáticas foram realizadas no conjunto de dados cujos valores eram os médios entre os obtidos experimentalmente. As mesmas foram feitas no software STATISTICA 8.0, com o qual foram determinados os parâmetros de cada modelo.

\section{RESULTADOS E DISCUSSÃO}

O aspecto físico dos grãos após estas secagens é apresentado na Figura 1.
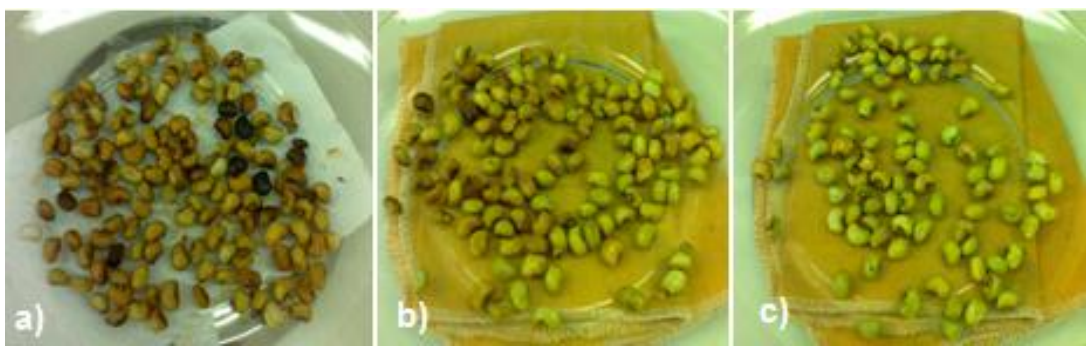

Figura 1 - Aspecto físico do feijão in naturaapós a secagem. Em a) tem-se Mi=50g, em b) $\mathrm{Mi}=40 \mathrm{~g}$ e em c) $\mathrm{Mi}=30 \mathrm{~g}$.

Com isso, a massa de $30 \mathrm{~g}$ foi adotada como a inicial, confirme foi dito anteriormente.

Os resultados dos modelos matemáticos aplicados no conjunto de dados cujos valores eram os médios entre os obtidos na condição mais adequada da secagem se encontram nas Tabelas 1 e 2 .

Tabela 1 - Valores dos parâmetros da secagem do feijão-verde in natura.

\begin{tabular}{cccccccccc}
\hline Modelos & $\mathbf{k}\left(\mathbf{s}^{-1}\right)$ & $\mathbf{n}$ & $\mathbf{A}$ & $\mathbf{c}$ & $\mathbf{L}$ & $\mathbf{R}^{\mathbf{2}}$ & $\mathbf{M B E}$ & $\mathbf{R M S E}$ & $\chi^{\mathbf{2}}$ \\
\hline Page & 0,182208 & 1,137452 & - & - & - & 0,996741 & 0,004924 & 0,070172 & 0,000358 \\
Lewis & 0,230545 & - & - & - & - & 0,992885 & 0,006693 & 0,081811 & 0,000733 \\
H.\& Pabis & 0,237107 & - & 1,030305 & - & - & 0,993864 & 0,008810 & 0,093860 & 0,000674 \\
Page Mod. & 0,223824 & 1,137510 & - & - & - & 0,996741 & 0,004929 & 0,070205 & 0,000358 \\
ESDF & - & - & 1,030352 & 0,187720 & 0,889736 & 0,993864 & 0,008801 & 0,093813 & 0,000723 \\
\hline
\end{tabular}

Tabela 2 - Valores dos parâmetros da secagem do feijão-verde com DO.

\begin{tabular}{cccccccccc}
\hline Modelos & $\mathbf{k}\left(\mathbf{s}^{-1}\right)$ & $\mathbf{n}$ & $\mathbf{A}$ & $\mathbf{c}$ & $\mathbf{L}$ & $\mathbf{R}^{\mathbf{2}}$ & $\mathbf{M B E}$ & $\mathbf{R M S E}$ & $\chi^{\mathbf{2}}$ \\
\hline Page & 0,109752 & 1,633247 & - & - & - & 0,999301 & 0,000594 & 0,024377 & 0,000099 \\
Lewis & 0,274376 & - & - & - & - & 0,951003 & 0,004739 & 0,068838 & 0,0064600 \\
H. \& Pabis & 0,305938 & - & 1,132028 & - & - & 0,967425 & 0,017791 & 0,133382 & 0,004601 \\
Page Mod. & 0,224074 & 1,224451 & & - & - & 0,951003 & 0,049783 & 0,223121 & 0,005803 \\
ESDF & - & - & 1,132064 & 3,354309 & 3,311226 & 0,967425 & 0,017806 & 0,133439 & 0,004955 \\
\hline
\end{tabular}


Com os resultados das Tabelas anteriores, obtiveram-se os gráficos das cinéticas das secagens (Figuras 2 e 3 ).

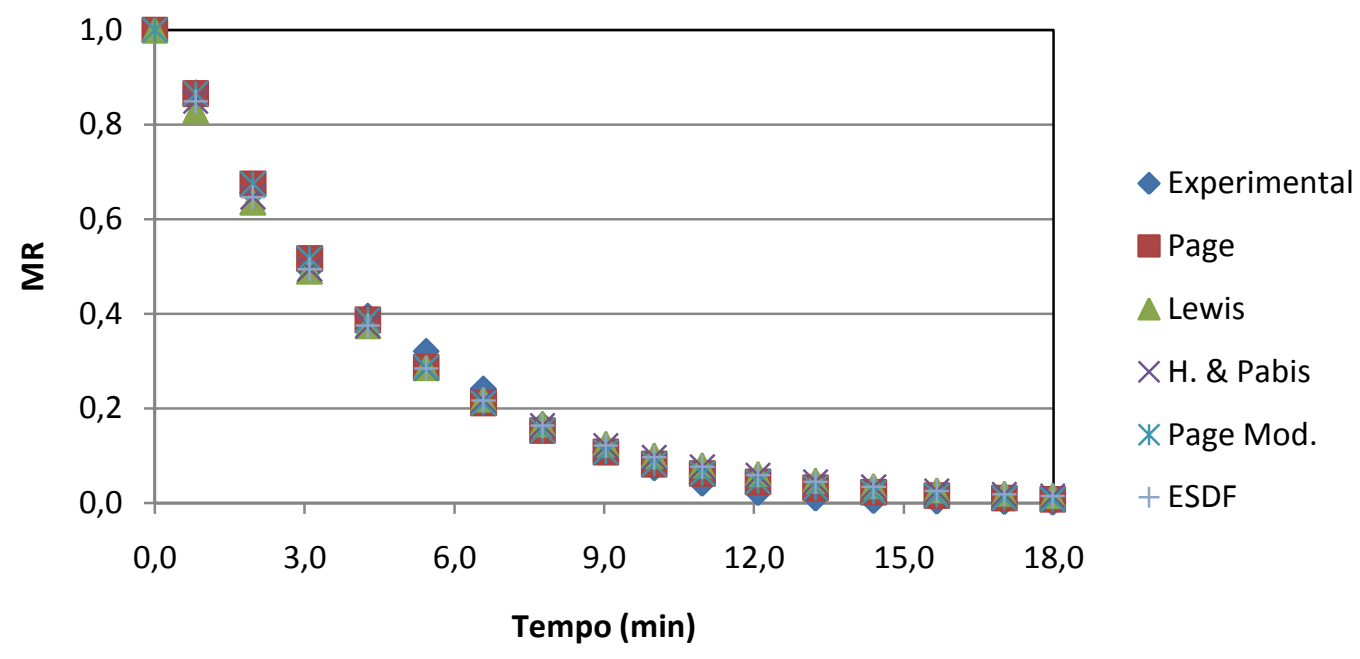

Figura 2 - Cinética da secagem para amostras in natura

Para as amostras in natura, as curvas dos modelos apresentaram valores próximos aos experimentais, indicando que todos obtiveram bons ajustes. Isto também é comprovado pelos valores dos parâmetros estatísticos da Tabela 1, os quais ficaram próximos dos ideais.

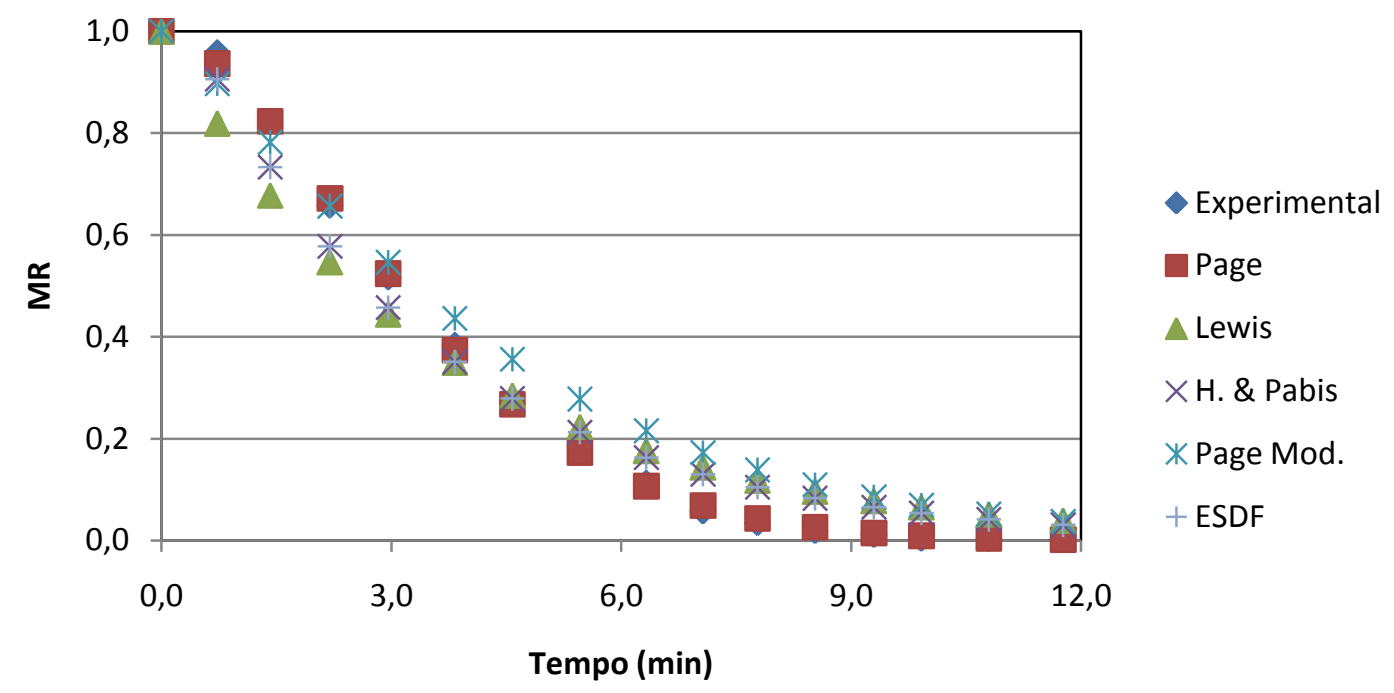

Figura 3 - Cinética da secagem para amostras com DO

Uma observação importante entre a secagem desses dois tipos de amostras, in natura e com OD, é que o tempo de secagem para as amostras que passaram pelo pré-tratamento, desidratação osmótica, mostrou-se menor, indicando uma economia energética e maior eficiência do processo.O tempo, em média, gasto para a secagem do feijão verde in naturafoi 
de dezessete minutos e para as amostras que passam pela desidratação osmótica foi de onze minutos, uma redução considerável.

O modelo mais adequado para a secagem do feijão verde em forno micro-ondas é aquele que apresenta maior valor de $\mathrm{R}^{2}$ e menores valores de MBE, RMSE e $\chi^{2}$. Desta forma, tanto para a modelagem da secagem do feijão verdein naturaquantopara a modelagem da secagem do feijão verde com desidratação osmótica, o modelo mais adequado foi o de Page.

Page descreve a secagem de grãos pela Equação 1:

$$
M R=\exp \left(-k \cdot\left(t^{n}\right)\right)
$$

Na qual o $n$ é um parâmetro adimensional de secagem e $k$ é a constante de secagem, $\mathrm{s}^{-1}$.

Outros autores também mostraram que o modelo de Page se mostrou mais adequado para diversos produtos da agricultura (White et al., 1981; Soares, 1986; Ramos et al., 1993/1994; Christ, 1996).

\section{CONCLUSÕES}

Dentre os modelos ajustados aos dados da cinética de secagem do feijão verde em forno micro-ondas, o tradicional modelo de secagem de Page é o que melhor descreve esta operação, com ajustes superiores a 0,99 para ambos os casos estudados (com e sem desidratação osmótica).

A partir do estudo realizado, pode-setambém notar a importância da realização da desidratação osmótica do feijão verde antes da prática da secagem utilizando a tecnologia de micro-ondas, pois esse pré-tratamento significa uma relevante redução no tempo de secagem das amostras, indicando uma economia energética e maior eficiência do processo.

\section{REFERÊNCIAS}

AZOUBEL, P.M.; AMORIM, M. R.; OLIVEIRA, S. S. B.; BAIMA, A.M.; CASTRO, M. R. Cinética de secagem de manga com e sem pré-tratamento ultrassônico. XVII COBEQ. FOZ DO IGUAÇU-PR, 2010.

CHRIST, D. Curvas de equilíbrio higroscópico e de secagem da canola (Brassica napus L. var. oleifera) e efeito da temperatura e da umidade relativa do ar de secagem sobre a qualidade das sementes. Viçosa: Universidade Federal de Viçosa, 1996. 50p. Dissertação Mestrado.

DA LUZ, C.; BAUDET, L.; FRANDOLOSO, V. Determinação do teor de água de sementes de arroz por secagem com microondas. Revista Brasileira de Sementes. v.20, n.1, p.70-74, 1998.

FENG, H.; TANG, J. Microwave Finish drying of diced apples in a spouted bed. J. of Food Science, 63, 679-683, 1998. 
GUNHAN, T.; DEMIR,V.; HANCIOGLU,E.; HEPBASLI,A. Mathematical modelling of drying of bay leaves. Energy Conversion and Management, 46, 1667-1679, 2005.

KROKIDA, M.K.; PHILIPPOPOULOS, C. Rehydration of Dehydrated Foods. Drying Technology, 23:4, $799-830,2005$.

MASKAN, M. Drying, shrinkage and rehydration characteristics of kiwifruits during hot air and microwave drying. J. Food Eng. 48, 177-182, 2001.

MENEZES, M.L.; KUNZ, C.C; PERINE, P.; PEREIRA, N.C. SANTOS, O. A. A; BARROS, T.D. Estudo da secagem convectiva do bagaço do maracujá amarelo. XVII COBEQ. FOZ DO IGUAÇU-PR, 2010.

MOREIRA, R.; CHENLO, F.; CHAGURI, L., C. Fernandes. Water absorption, texture, and color kinetics of air-dried chestnuts during rehydration. J. of Food Eng., 86, 584-594, 2008.

OLIVEIRA, L.G.; QUEIROGA, P.V.D.M.; BEZERRA, H.C.N.; MATA, W.; MATA, A.L.M.L. Medidas das propriedades dielétricas do feijão verde (Vigna unguiculata L. Walp) desidratado osmoticamente. XX CIC. Natal - RN, 2009.

PIOTROWSKI, D.; LENART, A., \& WARDZYSKI, A. Influence of osmotic dehydration on microwave-convective drying of frozen strawberries. J. of Food Eng., 65(4), 519-525, 2004.

RAMOS, A.M.; PEREIRA, J.A.M.; QUEIROZ, D.M. Equações de secagem de trigo em camada fina e determinação dos coeficientes de difusão de líquido. Revista Brasileira de Armazenamento, Viçosa, v.18-19, n.1-2, p.43-47, 1993/1994.

RASTOGI, N.K.; NAYAK, C.A.; RAGHAVARAO, K.S.M.S. Influence of osmotic pretreatments on rehydration characteristics of carrots. J. of Food Eng., 65, 287-292, 2004.

SANJUÁN, N.; SIMAL, S.; BON, J.; MULET, A. Modelling of broccoli stems rehydration process. J. of Food Eng. 42, 27-31, 1999.

SANTOS, L.D.M.; PRADO, M.M. Estudo da secagem de moringa oleifer. XVII COBEQ. FOZ DO IGUAÇU-PR, 2010.

SOARES, J.B. Curvas de secagem em camada fina e propriedades físicas de soja (Glicine max L.). Viçosa: Universidade Federalde Viçosa, 1986. 91p. Dissertação Mestrado.

WHITE, G.M.; BRIDGES, T.C.; LOEWER, O.J.; ROSS, I.J.Thin-layer drying model for soybeans.Transaction of the ASAE, St. Joseph, v.24, n.6, p.1643-1646, 1981. 